\title{
Production of platinum radioisotopes at Brookhaven Linac Isotope Producer (BLIP)
}

\author{
Suzanne V. Smith ${ }^{1,2, a}$, Elizabeth McCutchan ${ }^{3}$, Gülhan Gürdal ${ }^{4}$, Christopher Lister ${ }^{5}$, Lisa Muench ${ }^{1}$, Michael Nino ${ }^{3}$, \\ Alexandro Sonzogni ${ }^{3}$, Michal Herman ${ }^{3}$, Gustavo Nobre ${ }^{3}$, Chris Cullen ${ }^{1}$, Thomas Chillery ${ }^{5}$, Partha Chowdury ${ }^{5}$, \\ and Robert Harding ${ }^{5}$ \\ 1 Collider-Accelerator Department, Brookhaven National Laboratory, Upton NY 11973, USA \\ 2 Idaho State University, Idaho Accelerator Center, Department of Physics, Nuclear \& Electrical Engineering, Pocatello ID 83209, USA \\ 3 National Nuclear Data Center, Brookhaven National Laboratory, Upton NY 11973, USA \\ ${ }^{4}$ Physics Department, Millsaps College, Jackson MS 39202, USA \\ 5 Department of Physics and Applied Physics, University of Massachusetts, Lowell MA 01854, USA
}

\begin{abstract}
The accelerator production of platinum isotopes was investigated at the Brookhaven Linac Isotope Producer (BLIP). In this study high purity natural platinum foils were irradiated at 53.2, 65.7, 105.2, 151.9, 162.9 and 173.3.MeV. The irradiated foils were digested in aqua regia and then converted to their hydrochloride salt with concentrated hydrochloric acid before analyzing by gamma spectrometry periodically for at least 10 days post end of bombardment. A wide range of platinum (Pt), gold ( $\mathrm{Au}$ ) and iridium (Ir) isotopes were identified. Effective cross sections at BLIP for Pt-188, Pt-189, Pt-191 and Pt-195m were compared to literature and theoretical cross sections determined using Empire-3.2. The majority of the effective cross sections $(<70 \mathrm{MeV})$ confirm those reported in the literature. While the absolute values of the theoretical cross sections were up to a factor of 3 lower, Empire 3.2 modeled thresholds and maxima correlated well with experimental values. Preliminary evaluation into a rapid separation of Pt isotopes from high levels of Ir and Au isotopes proved to be a promising approach for large scale production. In conclusion, this study demonstrated that with the use of isotopically enriched target material accelerator production of selected platinum isotopes is feasible over a wide proton energy range.
\end{abstract}

\section{Introduction}

Approximately $50 \%$ of cancer patients receive platinum agents. They are used to treat a wide range of cancers alone and or in combination with other chemotherapeutic or biological agents such as antibodies. Their mechanism of action is similar however they have different side effects and activities against various cancers. Despite their wide spread use, there continues to be interest to gain a better understanding how to improve their efficacy and reduce side effects [1].

Personalized medicine is the use of screening methods to monitor the biological profile of a drug in a patient prior to administering therapeutic dose. For example, this can be performed by administering cisplatin and monitoring for segments of DNA that bond to the cisplatin to determine cellular responses [2]. A product under development for such as applications is PlatinDx [3]. Alternatively radiolabelled analogues of chemotherapeutic agents such as cisplatin can be used as theranostic agents which combine diagnostic and therapeutic functions into a single pharmaceutical. Using this approach, one can image the patient and determine uptake, identify responders to treatment, optimize dose and reduce side effects for the patient [4-7].

\footnotetext{
${ }^{a}$ e-mail: Suzanneoznq@gmail.com
}

Pt-195m $\left(\mathrm{T}_{1 / 2}=4\right.$ days $)$ has been produced at a number reactor sites and its incorporation to produce such radiopharmaceuticals as ${ }^{195 \mathrm{~m}} \mathrm{Pt}$-cisplatin and ${ }^{195 m} \mathrm{Pt}$-carboplatin has been demonstrated [4]. Recently, ${ }^{195 m}$ Pt-cisplatin produced using the high flux reactor position at SAFARI, South Africa was used in a phase 0 clinical trial to determine biological profile in healthy individuals $[6,7]$.

While Pt-195m has an attractive half-life and auger emissions that are ideal for therapy, its reactor production route yields products of moderate specific activity (i.e., $\approx 20 \mathrm{MBq} / \mathrm{mg}$ ) [7]. Therefore accelerator production of a wider selection of platinum radioisotopes with higher specific activities, physical emissions and half-lives is of growing interest for applications in theranostic agents for personalized medicine [8].

Cross sections for the accelerator proton-induced nuclear reactions on natural platinum have been reported [9]. Data is available up to $70 \mathrm{MeV}$ and show cross sections are suitable for production of ${ }^{191} \mathrm{Pt}\left(\mathrm{T}_{1 / 2}=2.802 \mathrm{~d}\right)$ via ${ }^{\text {nat }} \mathrm{Pt}(\mathrm{p}, \mathrm{pxn})^{191} \mathrm{Pt}$, ranging from approximately $8 \mathrm{mb}$ to $20 \mathrm{mb}$ for energies from 19 to $30 \mathrm{MeV}$ and broad peak at 40 to $65 \mathrm{MeV}$ with a high cross section $(\approx 400 \mathrm{mb})$. For the production of ${ }^{195 \mathrm{~m}} \mathrm{Pt}$, the ${ }^{\text {nat }} \mathrm{Pt}(\mathrm{p}, \mathrm{pxn}){ }^{195 \mathrm{~m}} \mathrm{Pt}$ cross sections vary from $7 \mathrm{mb}$ at $20 \mathrm{MeV}$ up to $45 \mathrm{mb}$ at $65 \mathrm{MeV}$. The cross section data suggest that the production of ${ }^{188} \mathrm{Pt}\left(\mathrm{T}_{1 / 2}=10.2 \mathrm{~d}\right)$ will increase at higher proton

(C) The Authors, published by EDP Sciences. This is an Open Access article distributed under the terms of the Creative Commons Attribution License 4.0 (http://creativecommons.org/licenses/by/4.0/). 
energies while the production of ${ }^{189} \mathrm{Pt}$, a relatively short lived isotope $\left(\mathrm{T}_{1 / 2}=10.87 \mathrm{hr}\right)$ shows promise at proton energies $>50 \mathrm{MeV}$, continuing to increase to $350 \mathrm{mb}$ at $70 \mathrm{MeV}$. Ref. [9] also propose that the indirect production route for Pt-189, Pt-188, Pt-191 via respective their Au parents is significantly higher than the direct route and as such are worthy of further investigation.

The present study examines the accelerator production of platinum isotopes across a range of proton energies and the predictive strength of the nuclear modeling code Empire-3.2 to assist in focusing research and production efforts [10]. It also reports a preliminary investigation into chemistry to isolate the Pt isotopes from large quantities of contaminating co-produced Ir and $\mathrm{Au}$ isotopes. Collectively the data will be used to determine commercially viable approaches (including choice of enriched target materials) to large-scale accelerator production of ${ }^{191} \mathrm{Pt},{ }^{195 \mathrm{~m}} \mathrm{Pt},{ }^{188} \mathrm{Pt}$ and ${ }^{189} \mathrm{Pt}$ at high proton energies.

\section{Materials and methods}

Chemicals used in the study were of analytical grade. Platinum foils were obtained from Goodfellow. They were of high purity (> 99.99\%) and $0.025 \mathrm{~mm}$ thick. The foils were cut to fit within custom made aluminum (Alloy 6061) 8 screw bolted can fitted with a target well (diam. $29.21 \mathrm{~mm}$ ). The back of the target can is cut away to allow for water cooling of the target. The target cans were sealed with an Inconel c-ring under nitrogen and then vacuum tested to ensure no nitrogen gas leaks.

The target station at BLIP is beneath ground and watercooled. It receives direct pulses from the linac typically at $117 \mathrm{MeV}$ which is used for the production of ${ }^{82} \mathrm{Sr}$. Two target assembly boxes and incremental tuning of the entry proton energy (i.e., 37, 66, 93, 117, 139, 160, 181, and $200 \mathrm{MeV}$ ) up to $200 \mathrm{MeV}$ protons allows for multiple targets to be irradiated and a range of target arrays to be investigated. The proton beam from the Linac is projected through a series of windows of varying thickness (i.e., beryllium-copper $(0.3048 \mathrm{~mm}))$, AlBemet $^{\mathrm{s}}(0.3048 \mathrm{~mm})$, stainless steel $(0.787 \mathrm{~mm}), 2.67 \mathrm{~mm}$ of cooling water, stainless steel target box window $(0.508 \mathrm{~mm})$ and a layer of cooling water before reaching the target assembly box. To achieve desired proton energies on the platinum foils, target arrays were designed with specified metal degraders (usually aluminum or stainless steel) and at least $5.08 \mathrm{~mm}$ water gaps between layers to ensure adequate cooling. Energy degradation for each layer was calculated using TRIM subroutine in SRIM 2013 for each array [11]. Entry energy to target arrays (either 198.8 or $116.8 \mathrm{MeV}$ ) was determined by a laser profile monitor and the total accumulated charge during target irradiation was measured using a beam current transformer located in the beam line of the linac. The projection of beam spot area was varied using activated aluminum foils. The total number of platinum foils irradiated, their thickness and weight, the entry proton energy and estimated current on each foil is given in Table 1.

Thermal properties of platinum are attractive for high energy proton irradiations. Thermal conductivity $\left(71.6 \mathrm{~W} \cdot \mathrm{m}^{-1} \cdot \mathrm{K}^{-1}\right)$, melting $\left(1768.8^{\circ} \mathrm{C}\right)$ and boiling $\left(3825^{\circ} \mathrm{C}\right)$ points are all high. The heat profile of the platinum target within an aluminum can was calculated
Table 1. Experimental parameters for proton irradiations on Platinum targets including incident beam energy, target thickness and beam current.

\begin{tabular}{|c|c|c|}
\hline $\begin{array}{c}\text { Energy } \\
(\mathbf{M e V})\end{array}$ & $\begin{array}{c}\text { Thickness of } \\
\text { Foil }(\mathbf{m m})\end{array}$ & $\begin{array}{c}\text { Estimated current } \\
\text { on target }(\boldsymbol{\mu} \mathbf{A})\end{array}$ \\
\hline 173.3 & 0.025 & 96.0 \\
\hline 162.9 & 0.025 & 96.0 \\
\hline 151.9 & 0.025 & 96.0 \\
\hline 105.2 & 0.025 & 23.8 \\
\hline 65.7 & 0.025 & 115.3 \\
\hline 53.2 & 0.025 & 115.2 \\
\hline
\end{tabular}

with the software program ANSYS. Beam characteristics were set with the instantaneous current of $40 \mathrm{~mA}$, bunchwidth of $0.000425 \mathrm{sec}$, frequency of $6.667 \mathrm{~Hz}$ and protons of $1.061 \times 10^{14}$. ANSYS finite element model (parameters: 2D axi-symmetric model, steady state analysis, thermal contact resistance between layers, steady state water cooling convection on exterior and gaussian internal heat generation for all bodies-aluminum contact resistance between can and platinum target) estimated maximum temperature for platinum target foil and aluminum can in a typical irradiation at BLIP would be no more than $268{ }^{\circ} \mathrm{C}$, and $223{ }^{\circ} \mathrm{C}$, respectively; well below respective melting points of $1116^{\circ} \mathrm{C}$ and $582^{\circ} \mathrm{C}$.

\subsection{Gamma spectrometry}

Typically the aluminum can containing the platinum foil was allowed to decay for up to 24 hours before transporting to production facilities and opening. The platinum foils $(0.025 \mathrm{~mm})$ were dissolveded in boiling aqua regia $(10 \mathrm{~mL})$ and then evaporated to dryness repeatedly with concentrated hydrochloric acid $(\mathrm{HCl})$ $(2 \mathrm{~mL} \times 3)$ to remove any nitrate ions. In each case the final residue were dissolved in $10 \mathrm{~mL}$ of $0.1 \mathrm{M} \mathrm{HCl}$ and then accurately serially diluted for analysis on the gamma spectrometer ensuring dead time remained below $10 \%$.

Each sample was assayed on unsuppressed (ORTEC HPGe detector $\left(\right.$ AMETEK $^{\mathrm{s}}$ ) Oak Ridge, TN) repeatedly at BNL. Selected samples were also analyzed with a $40 \%$ relative efficiency ORTEC HPGe detector surrounded by BGO scintillator to enable Compton-suppression for gamma emission assignment at U. Mass Lowell. The GammaVision-32 software, version 6.01 was used for spectra acquisition and processing. The standards were purchased from Eckert and Zieglers (Atlanta, GA). The Gamma-Vision spectra were converted into RADWARE format [12] and analyzed using the GF3 package. Gamma emissions from each sample were assessed multiple times (at least 5 spectra) daily on standard gamma spectrometers for at least one week and in some cases intermittently for a further 17 days. Spectra were analyzed for isotopes such

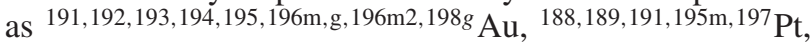
and ${ }^{186,188,189,190,192,194 \mathrm{~m}}$ Ir as well as their decay daughters. Gammas in each spectra were monitored for half-life and then assigned to the respective radioisotopes. Table 2 . gives a list of selected $\mathrm{Au}$ and $\mathrm{Pt}$ isotopes verified in samples. Many of the gamma ray transitions for the Pt, $\mathrm{Au}$ and Ir isotopes produced were overlapping in energy. Care was taken to identify unique gammas for use in the determination of the effective cross sections for each isotope. 
Table 2. Radioisotopes identified and their decay characteristics.

\begin{tabular}{|c|c|c|}
\hline Isotope & Half-Life & $\begin{array}{c}\text { Gammas (Intensity)* } \\
\operatorname{keV}(\%)\end{array}$ \\
\hline Pt-188 & $\begin{array}{l}10.2 \\
\text { days }\end{array}$ & $\begin{array}{c}187.6(19.4), \mathbf{1 9 5 . 1} \\
\text { (18.6), } 381.4(7.5) \\
423.5(4.4)\end{array}$ \\
\hline Pt-189 & 10.87 hours & $\begin{array}{c}243.5(7.0), 568.9(7.1), \\
721.4(9.3)\end{array}$ \\
\hline Pt-191 & 2.802 days & $\begin{array}{c}96.5(3.3), 129.4(3.2), \\
172.2(3.7), 351.2(3.4), \\
359.9(6.0), 409.4(8.0), \\
\mathbf{5 3 8 . 9}(\mathbf{1 3 . 7})\end{array}$ \\
\hline Pt-195m & 4.02 days & 98.9(11.4), $129.8(2.8)$ \\
\hline $\mathrm{Au}-191 \mathrm{~g}$ & 3.18 hours & $\begin{array}{c}277.9(7.0), 399.8(4.7) \\
478.0(3.9), \mathbf{5 8 6 . 4} \\
(\mathbf{1 7 . 0})\end{array}$ \\
\hline Au-192 & 4.94 hours & $\begin{array}{c}296.0(22.0), 308.5 \\
(3.4), \mathbf{3 1 6 . 5}(\mathbf{5 8 . 0})\end{array}$ \\
\hline Au-196g & 6.183 days & $\begin{array}{c}333.03(22.9), 355.73 \\
(\mathbf{8 7 . 0})\end{array}$ \\
\hline Au-198g & 2.695 days & $411.8(95.6)$ \\
\hline
\end{tabular}

* Gamma emission used to calculate cross sections are bolded.

The cross section for each isotope was calculated using the standard cross section equation. Gamma-ray yields measured were determined for unique gamma emissions of the isotope from each gamma spectrum. Analysis took into consideration beam current, counting time for spectrum, target thickness, efficiency of the detector at the energy of interest and the absolute intensity of the gamma emission per parent nucleus together with the dilution factor related with the chemical separation for the sample used to measure the cross section. Systematic uncertainties relating to the beam flux, foil processing and detector efficiency are estimated as $10 \%$ overall. In addition, the gamma-ray spectrum analysis for each isotope introduces its own uncertainty relating to the peak fitting, potential doublet nature of transitions and uncertainty in absolute gamma-ray transition strength. These uncertainties are added in quadrature to the $10 \%$ systematic uncertainty in the final quoted values.

\subsection{EMPIRE calculations}

The Empire 3.2 code was used to calculate the cross sections for isotopes populated by proton bombardment of a natural platinum target. Since natural platinum has six stable isotopes [Pt-190 (0.01\%); Pt-192 (0.78\%); Pt-194 (32.97\%); Pt-195 (33.83\%), Pt-196 (25.24\%) Pt$198(7.16 \%)]$ the total cross sections were determined by calculating the cross section from each individual stable isotope, then summing all isotopes, weighted by their natural abundances. The code was run by taking the standard recommended input parameters for all possible nuclei which could be populated by the nuclear reactions, including the Exciton and Hybrid Monte Carlo preequilibrium models and compared with the experimental results [10].

The level densities for each compound nucleus were adjusted based on the experimental level scheme. Figures 1 to 4 compare the experimental cross sections from the literature [9] and at BLIP with those calculated using Empire 3.2 for Pt-188, Pt-189, Pt-191, Pt-195m. The

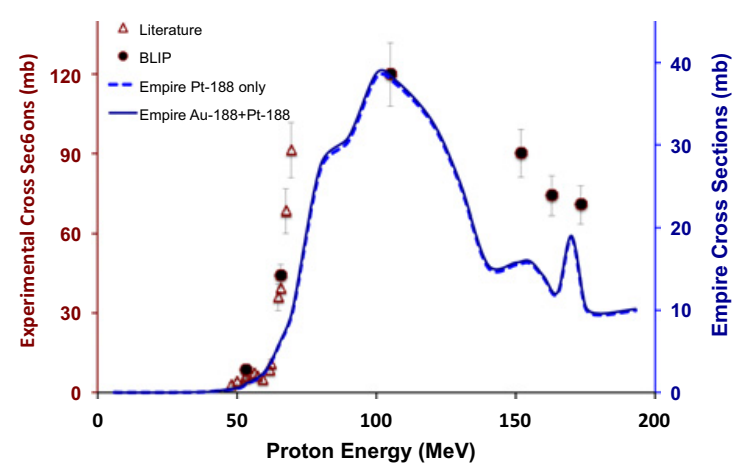

Figure 1. Cross Sections for Production of Pt-188 from Natural Platinum. (Note that the right and left y axes are plotted with different scales.)

theoretical curves include with and without contributions from respective Au isotopes where relevant. Attempts were made to optimize the EMPIRE calculations focusing on the reproduction of the previously measured $<70 \mathrm{MeV}$ data from Ref. [9]. Calculations were performed replacing the default spherical potential with a deformed one. In addition, the pre-equilibrium parameters were varied. These adjustments did not alter the EMPIRE predictions significantly.

\subsection{Purification of platinum isotopes}

The irradiated foils were dissolved in boiling aqua regia, evaporated to dryness and then repeatedly digested with concentrated $\mathrm{HCl}$ to ensure conversion of platinum species to their chloride salt. The Pt radionuclides were isolated as the platinum diaqua ammonia species in a similar manner to that described previously [5]. The residue was dissolved in $0.1 \mathrm{M} \mathrm{HCl}$ and the platinum hexachloride species was converted to ammonium iodide salt with the addition of potassium iodide and careful addition of ammonia. The precipitation of the $\mathrm{AgCl}$ from the addition of silver nitrate afforded conversion of the soluble $\left(\mathrm{Pt}\left(\mathrm{NH}_{3}\right)_{2}\left(\mathrm{H}_{2} \mathrm{O}\right)_{2}\left(\mathrm{NO}_{3}\right)_{2}\right.$.

\section{Results and discussion}

A large number of gold $(\mathrm{Au})$, platinum $(\mathrm{Pt})$ and Iridium (Ir) isotopes were produced when high purity natural platinum foils (with six stable isotopes) were bombarded with protons in the energy range from 53.2 to $173.3 \mathrm{MeV}$ at BLIP. Spectra were quite complex and separation and identification of multiple unique gamma transititons for an isotope was not always possible for use in the quantification of accurate effective cross sections. A combination of Compton suppressed and unsuppressed gamma spectrometry along with mapping of gamma lines half lives were used to confirm assignment of gamma emissions. The spectra were analyzed using two software programs (Gamma-Vision and GF3 package). The BLIP cross sections for Pt-188, Pt-189, Pt-191 and Pt-195m are compared with cross sections reported in the literature [9] and those calculated using Empire-3.2 (see Figs. 1-4).

Pt-188 has a 10.2 day half-life and gamma emissions that are suitable for in vitro and in vivo pre-clinical biodistribution and imaging studies.

The gamma emission at $195.1 \mathrm{keV}$ is $18.6 \%$ abundant and ideal for the quantification of the $\mathrm{Pt}-188$ production 


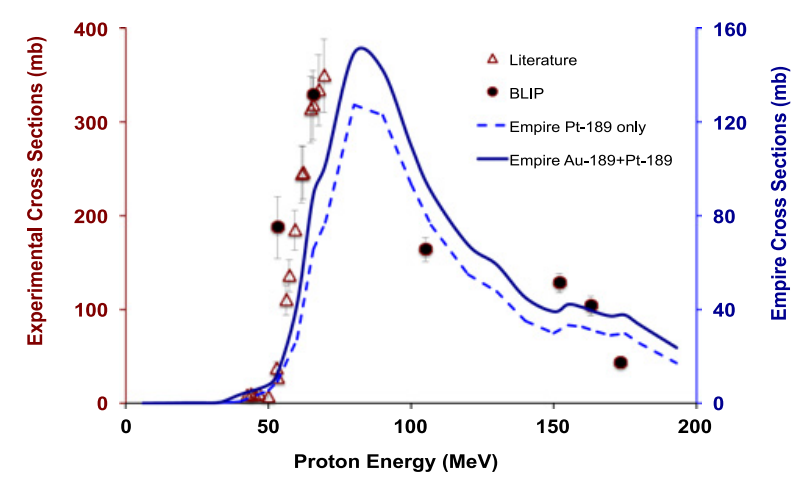

Figure 2. Cross Sections for Production of Pt-189 from Natural Platinum. (Note that the right and left y axes are plotted with different scales.)

and calculation of the effective cross section at BLIP. Figure 1 illustrates the literature cross section and effective cross section at BLIP for various proton energies. There is excellent agreement between the literature and BLIP cross sections at both 53 and $66 \mathrm{MeV}$. The effective cross sections at $\geqslant 105 \mathrm{MeV}$ are higher than those calculated with EMPIRE however the trend in cross sections over the $200 \mathrm{MeV}$ range correlates (i.e., thresholds and maxima) well with both sets of experimental cross section data. EMPIRE calculations indicate that Pt-194 and Pt-195 stable isotope are the main contributors to the direct production of Pt-188 at proton energies between 80 to $120 \mathrm{MeV}$.

Pt-189 is short lived radioisotope $\left(\mathrm{T}_{1 / 2}=10.87 \mathrm{hr}\right)$ that has potential for application in in vitro and metabolic studies. Pt-189 can be produced directly or indirectly from the decay of Au-189 isotopes. For this study the cross section for Pt-189 was determined after decay of the Au189 isotopes. The effective cross section for Pt-189 at $53 \mathrm{MeV}$ was measured to be $187 \mathrm{mb}$ while the respective literature value was only $27 \mathrm{mb}$ (see Fig. 2).

In contrast, at $66 \mathrm{MeV}$ the cross section appears to converge with values of $328.3 \mathrm{mb}$ and $317.8 \mathrm{mb}$ respectively for BLIP and literature values. Figure 2 also illustrates plots for Empire cross sections with and without contribution from Au-189. The data clearly show the contribution from Au-189 for Pt-189 values are particularly significant. The Empire plots have similar shape to the literature value at lower proton energies and good agreement on threshold and trends in maxima around $70-90 \mathrm{MeV}$. However the absolute values are approximately 3 to 4 fold lower across the energy range investigated. The EMPIRE results indicate that the sharp rise at $70 \mathrm{MeV}$ is most likely from the (p,xn) and p,pxn) reactions on the highly abundant Pt-194 and Pt-195. This is in agreement with predictions reported in the literature [9].

Pt-191 is a particularly interesting isotope for applications in personalized medicine. Its half life (2.8 days) and gamma emissions are well suited for SPECT imaging and the drug risk assessment. As spectulated in the literature Pt-191 can be produced by direct reactions on Pt targets and indirectly via the decay of Au-191. Figure 3 plots the effective cross sections (including Au191 contributions) for Pt-191 for samples irradiated at BLIP. These values are considerably lower that those reported at $53 \mathrm{MeV}$ in the literature and may be explained

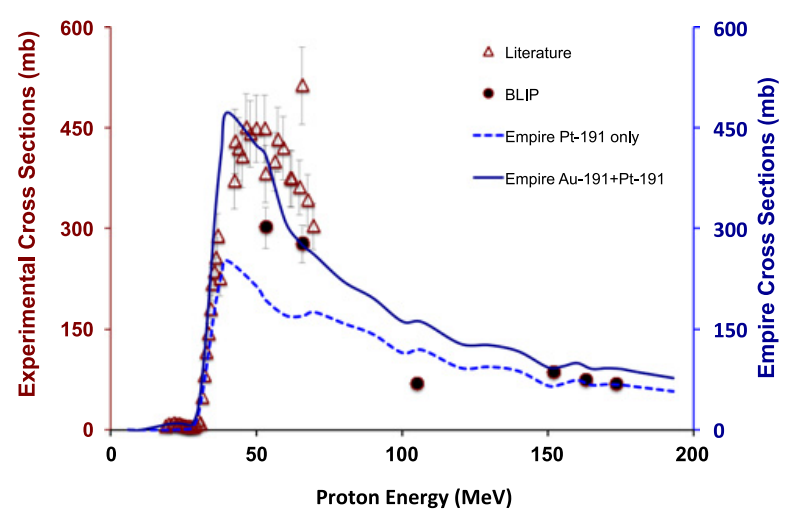

Figure 3. Cross Sections for Production of Pt-191 from Natural Platinum.

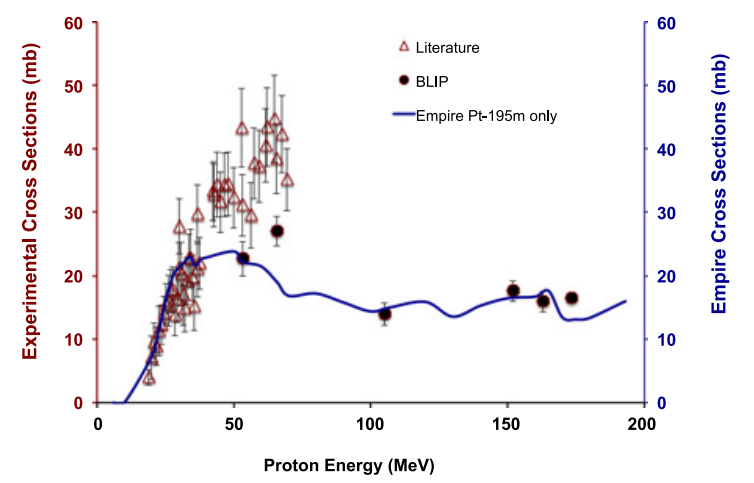

Figure 4. Cross Sections for Production of Pt-195m from Natural Platinum.

by the scatter and large associated errors in literature cross sections. Values obtained at $66 \mathrm{MeV}$ for BLIP appear to agree within error with the literature values.

In contrast with the Pt-188 and Pt-189 predictions Empire predictions for Pt-191 production agree well across the entire energy range investigated. Overall, we observe that the agreement of EMPIRE with the experimental data improves as the number of evaporated particles decreases. The rapid increase in production of Pt-191 over the 30 to $70 \mathrm{MeV}$ proton energy range is mostly likely due to contribution from Au-191 as well as some from (p,pxn) reaction on Pt-194, Pt-195 and Pt-196.

Pt-195m is the most studied platinum isotope in the literature. Readily produced in reactors, it has already been used as a theranostic agent, utilizing both its Auger electron emission for therapy and its gamma emission for imaging. The accelerator production provides for an alternative source for its production. Figure 4 illustrates both experimental cross section data from the literature and that determined in this work. It compares these values to the predicted EMPIRE cross section. Values obtained at BLIP are lower than those reported in the literature. This deviation could be explained by the efficiency of detector used. The $98.9 \mathrm{keV}$ transition is close to the point of inflection of the efficiency curve in a standard HPGe detector. The fit for EMPIRE data are consistent with literature initially $(<30 \mathrm{MeV})$. Effective cross sections determined at BLIP across the entire energy range agree well with EMPIRE data. Deviations in collected data from 30 to $70 \mathrm{MeV}$ suggests further investigation is required. 

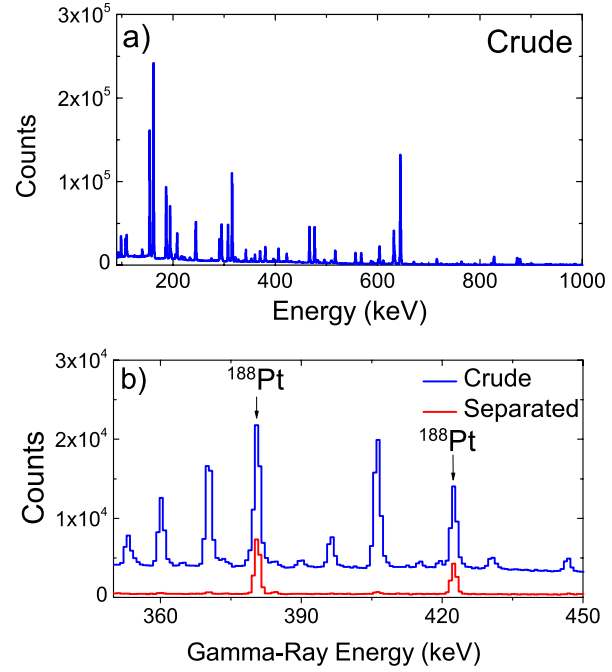

Figure 5. Gamma Spectra of (a) crude Pt foil and (b) crude and purified sample Pt-188 (expanded).

\subsection{Separation of platinum isotopes}

Figures 5(a) and 5(b) show the comparison of the gamma-ray spectrum of both crude platinum target and purified sample using literature reported methods. Once the target is digested the separation was complete within 1.5 hours. Samples were run on Compton suppressed gamma spectrometer to check for purity. Spectra clearly show the Pt-188 is very pure with additional signals from Ir-188 due to the decay of its parent Pt-188. This method shows promise for adaption to large-scale production.

\section{Conclusions}

Cross sections for the production of various Platinum isotopes via proton bombardment of natural platinum foils were investigated at BLIP. Overall the majority of the measured BLIP cross sections confirmed the literature values [9] at low proton energies, and provided new data points at high proton energies. The comparison of the experimental results with EMPIRE calculations for Pt-188, Pt-189, Pt-191 and Pt-195m showed good agreement overall for identifying threshold and maxima for proton energies for their production. Empire provided valuable information to guide investigations into large scale production of the four platinum isotopes and assist in the choice of enriched stable isotopes to use for optimizing production yield and purity.

U.S. DOE Office of Science, Office of Nuclear Physics and Isotope Program is acknowledged for funding: ST 50-01-02,
ST5001030, DE-FG02-94ER40848 and Contract Nos. DE-AC02-98CH10946, DE-AC02-06CH11357 and the DOE Office of Science, Office of Workforce Development for Teachers and Scientists (WDTS) under the Science Undergraduate Laboratory Internships Program (SULI). This work was supported in part by the U.S. Department of Energy, Office of Science, Office of Workforce Development for Teachers and Scientists (WDTS) under the Visiting Faculty Program (VFP).

\section{References}

[1] M. Galanski, M.A. Jakupec, B.K. Keppler, Curr Med Chem. 12, 2075-2094 (2005) T.C. Johnstone, G. Young Park and S.J. Lippard, Anticancer Res. 43(1), 471-476 (2014)

[2] G.D. Cimino, C. Pan and P.T. Henderson, Bioanalysis 5(3), 369-391 (2013)

[3] http://acceleratedmeddiagnostics.com/ platindx/

[4] J. Arberg, S. Bojrokman, L. Einarssar, B Frankenberg, H. Lundqvist, S. Mattsson, K. Norrgren, O Scheike and R. Wallin, Acta. Oncol. 38, 221-228 (1999)

[5] S.V Smith, Methods of synthesis and use of radiolabeled platinum chemotherapeutic agents. Patent WO 2001070755A1:Sep 27 (2001) K. Kawai, H. Maki, W. Ehrlich, M. Akaboshi, J Radioanal. Nucl. Chem. Lett. 136, 67-74 (1989) S.E. Buckley, A. Pa, C.J. Evans, A.M. El-Sharkawi, Phys. Med. Biol. 51(5), 1325-32 (2006)

[6] J.R. Zeevaart, J. Wagener, B. Marjanovic-Painter, M. Sathekge, N. Soni, C. Zinn, G. Perkins and S.V Smith. J Radiolabel Comp. 56(9-10), 495-503 (2013)

[7] M. Sathekge, J. Wagener, S.V. Smith, N. Soni, B. Marjanovic-Painter, C. Zinn, C. Van de Wiele, Y. D'Asseler, G. Perkins, J.R. Zeevaart. Nuklearmedizin 52(6), 222-227 (2013)

[8] 2015_NSACI_report. The 2015 Long Range Plan for the DOE-NP Isotope Program. http://science. energy.gov/ /media/np/nsac/pdf/docs/ 2015/2015_NSACI_Report_to_NSAC_Final .pdf

[9] A.F. Tarkanyi et al. Nucl. Instr. and Meth. in Phys. Res. B 226, 473-489 (2004)

[10] Empire-3.2 (Malta) Nuclear Reaction Code, M. Herman et al, Retrieved from https://www.nndc.bnl.gov/empire/main.html

[11] J.F. Ziegler, J.P. Biersack, The stopping and range of ions in matter (SRIM) http://www.srim.org (2013)

[12] D.C. Radford, Nucl. Instr. Meth. A 361, 297 (1995) 